\title{
Analytic Solutions of the Eigenvalues of Mathieu's Equation
}

\author{
Chein-Shan Liu ${ }^{1,2}$ \\ ${ }^{1}$ College of Mechanics and Materials, Hohai University, Nanjing, Jiangsu 210098, China \\ ${ }^{2}$ Center of Excellence for Ocean Engineering, Center of Excellence for the Oceans, National Taiwan Ocean University, \\ Keelung 202-24, Taiwan \\ Correspondence: Chein-Shan Liu, College of Mechanics and Materials, Hohai University, Nanjing, Jiangsu 210098, \\ China. E-mail: csliu@ntou.edu.tw; csliu58@163.com
}

Received: October 27, 2019 Accepted: November 20, 2019 Online Published: December 12, 2019

doi:10.5539/jmr.v12n1p1 URL: https://doi.org/10.5539/jmr.v12n1p1

\begin{abstract}
Mathieu's eigenvalue problem $-y^{\prime \prime}(x)+2 e_{0} \cos (2 x) y(x)=\lambda y(x), 0<x<\ell$ is symmetric if $\cos (2 x)=\cos (2 \ell-2 x)$ for $\ell=k_{0} \pi, k_{0} \in \mathbb{N}$, and skew-symmetric if $\cos (2 x)=-\cos (2 \ell-2 x)$ for $\ell=\pi / 2$. Two typical boundary conditions are considered. When the eigenfunctions are expanded by the orthonormal bases of sine functions or cosine functions, we can derive an $n$-dimensional matrix eigenvalue problem, endowing with a special structure of the symmetric coefficient matrix $\mathbf{A}:=\left[a_{i j}\right], a_{i j}=0$ if $i+j$ is an odd integer. Based on it, we can obtain the eigenvalues easily and analytically. When $\ell=k_{0} \pi, k_{0} \in \mathbb{N}$, we have $a_{i j}=0$ if $|i-j|>2 k_{0}$. Besides the diagonal band, $\mathbf{A}$ has two off-diagonal bands, and furthermore, a cross band appears when $k_{0} \geq 2$. The product formula, the recursion formulas of characteristic functions and a fictitious time integration method (FTIM) are developed to find the eigenvalues of Mathieu's equation.
\end{abstract}

Keywords: eigenvalues of Mathieu's equation, special matrix eigenvalue problem, product formula, recursion formula, fictitious time integration method (FTIM)

\section{Introduction}

Mathieu's equation is a well known second-order ordinary differential equation (ODE), endowing with periodic coefficient (McLachlan 1947; Bateman \& Erdelyi, 1955; Meixner, Schäfke \& Wolf, 1980). A number of physical phenomena and engineering problems can be described by Mathieu's equation, which also appears in the solution of the Helmholtz equation of an elliptic membrane by using the method of separation of variables (Plestenjak, Gheorghiu \& Hochstenbach, 2015). Mathieu's equation comes into the realm of mathematical physics for wave propagation, electromagnetic, elastic membrane and heat conduction, when there is some elliptic symmetry in the problems. Developing an analytic solution of Mathieu's equation is significant from the mathematical theoretical aspect (Gadella, Giacomini \& Lara, 2015).

In the paper we consider the following Mathieu's eigenvalue problem (Bujurke, Salimath \& Shiralashetti, 2008; Gheorghiu, Hochstenbach, Plestenjak \& Rommes, 2012):

$$
-y^{\prime \prime}(x)+q(x) y(x)=\lambda y(x), \quad 0<x<\ell,
$$

where $q(x)=2 e_{0} \cos 2 x$, and $2 e_{0}$ is a constant amplitude. We are going to study the eigenvalues for the following four problems:

$$
\begin{aligned}
& \text { Problem 1: } y(0)=y(\ell)=0, \quad \ell=\frac{\pi}{2}, \\
& \text { Problem 2: } y^{\prime}(0)=y^{\prime}(\ell)=0, \quad \ell=\frac{\pi}{2}, \\
& \text { Problem 3: } y(0)=y(\ell)=0, \quad \ell=k_{0} \pi, \quad k_{0}=1,2,3, \\
& \text { Problem 4: } y^{\prime}(0)=y^{\prime}(\ell)=0, \quad \ell=k_{0} \pi, \quad k_{0}=1,2,3 .
\end{aligned}
$$

For the first two problems and the last two problems we have, respectively,

$$
\begin{aligned}
& q(x)=-q(\ell-x)=2 e_{0} \cos (2 x), \\
& q(x)=q(\ell-x)=2 e_{0} \cos (2 x) .
\end{aligned}
$$

For Eq. (6), we have a skew-symmetric Mathieu's eigenvalue problem, while for Eq. (7), we have a symmetric Mathieu's eigenvalue problem. The coefficient matrices for Problems 1 and 2 are of special type tridiagonal Jacobi matrices. 
The paper is organized as follows. In Section 2, we transform Mathieu's eigenvalue problem to a matrix eigenvalue problem. In Section 3 for Problems 3 and 4 , the coefficient matrix is proved to have a special structure of $\mathbf{A}:=\left[a_{i j}\right]$, with $a_{i j}=0$ if $i+j$ is an odd integer. Problems 1 and 2 are solved in Section 4, where we develop a fictitious time integration method (FTIM) to obtain eigenvalues, based on the recursion formula to compute the characteristic function. More importantly, we develop a powerful product formula in Section 5 for Problems 3 and 4. Based on the product formula, the FTIM is used separately, to solve the odd characteristic equation and the even characteristic equation. The eigenvalues clustering phenomenon for Problem 3 is demonstrated in Section 6. The numerical methods to obtain the eigenvalues by using the FTIM are carried out for Problem 3 in Section 6, and for Problem 4 with different $k_{0}$ in Section 7. Finally, some conclusions are drawn in Section 8.

\section{Transforming to a Matrix Eigenvalue Problem}

Let

$$
y(x)=\sum_{k=1}^{n} x_{k} \phi_{k}(x)
$$

be the eigenfunction, where $x_{k}$ are coefficients to be determined, and $\phi_{k}(x)$ are trial functions, satisfying the same boundary conditions for $y(x)$.

For the boundary conditions $y(0)=y(\ell)=0$ the simplest orthonormal bases are

$$
\phi_{k}(x)=\sqrt{\frac{2}{\ell}} \sin \frac{k \pi x}{\ell},
$$

while for the boundary conditions $y^{\prime}(0)=y^{\prime}(\ell)=0$ the simplest orthonormal bases are

$$
\phi_{k}(x)=\sqrt{\frac{2}{\ell}} \cos \frac{k \pi x}{\ell} .
$$

Multiplying both the sides of Eq. (1) by $\phi_{j}(x)$, integrating it by parts from $x=0$ to $x=\ell$ and using the corresponding boundary conditions, we can derive

$$
\int_{0}^{\ell}\left[y^{\prime}(x) \phi_{j}^{\prime}(x)+q(x) y(x) \phi_{j}(x)\right] d x=\lambda \int_{0}^{\ell} y(x) \phi_{j}(x) d x .
$$

Inserting Eq. (8) for $y(x)$ into Eq. (11), taking $j=1, \ldots, n$, and using the orthonormality of $\phi_{j}(x)$, renders an $n$-dimensional eigenvalue problem:

$$
\mathbf{A x}=\lambda \mathbf{x},
$$

where $\mathbf{x}=\left(x_{1}, \ldots, x_{n}\right)^{\mathrm{T}}$ is an $n$-dimensional eigenvector, and simultaneously the components $a_{j k}$ of the symmetric coefficient matrix $\mathbf{A}$ are given by

$$
a_{j k}:=\int_{0}^{\ell}\left[\phi_{j}^{\prime}(x) \phi_{k}^{\prime}(x)+q(x) \phi_{j}(x) \phi_{k}(x)\right] d x, j, k=1, \ldots, n,
$$

where $a_{j k}=a_{k j}$. Upon solving the above matrix eigenvalue problem (12), we can obtain the eigenvalues $\lambda_{j}, j=1, \ldots, n$ of Eq. (12).

\section{Special Matrices}

Problem 1 endows a simple structure of the coefficient matrix with the components $a_{i j}$ given by

$$
\text { Problem 1: } a_{i j}= \begin{cases}(2 i)^{2} & \text { if } i=j \\ e_{0} & \text { if } i-j= \pm 1 .\end{cases}
$$

Hence, $\mathbf{A}=\left[a_{i j}\right]$ is a three bands tridiagonal matrix.

For problem 2, we have

$$
\mathbf{A}=\left[\begin{array}{ccccccccc}
0 & \sqrt{2} e_{0} & 0 & 0 & \ldots & 0 & 0 & 0 & 0 \\
\sqrt{2} e_{0} & 2^{2} & e_{0} & 0 & \ldots & 0 & 0 & 0 & 0 \\
\vdots & \vdots & \vdots & \vdots & \ldots & \vdots & \vdots & \vdots & \vdots \\
0 & 0 & 0 & 0 & \ldots & e_{0} & 2^{2}(n-3)^{2} & e_{0} & 0 \\
0 & 0 & 0 & 0 & \ldots & 0 & e_{0} & 2^{2}(n-2)^{2} & e_{0} \\
0 & 0 & 0 & 0 & \ldots & 0 & 0 & e_{0} & 2^{2}(n-1)^{2}
\end{array}\right]
$$


Let us consider Problem 3 with $k_{0}=1$, of which the components $a_{i j}$ are given by

$$
\text { Problem 3: } a_{i j}= \begin{cases}1-e_{0} & \text { if } i=j=1, \\ i^{2} & \text { if } i=j \geq 2, \\ e_{0} & \text { if } i-j= \pm 2 .\end{cases}
$$

$\mathbf{A}=\left[a_{i j}\right]$ with $a_{i j}=a_{j i}$ is a three bands symmetric matrix. Notice that $i-j= \pm 2$ is different from that $i-j= \pm 1$ in Eq. (14).

For Problem 3 with $k_{0} \geq 2$, it is more complex with

$$
\text { Problem 3: } a_{i j}= \begin{cases}\left(\frac{i}{k_{0}}\right)^{2} & \text { if } i=j, i+j \neq 2 k_{0} \\ 1-e_{0} & \text { if } i=j, i+j=2 k_{0} \\ e_{0} & \text { if } i-j= \pm 2 k_{0} \\ -e_{0} & \text { if } i+j=2 k_{0}, i \neq j\end{cases}
$$

Upon comparing with Eq. (16), a new cross band appears due to the last term occurring when $i+j=2 k_{0}, k_{0} \geq 2$.

For problem 4 with $k_{0} \geq 1$, we have

$$
\text { Problem } 4: a_{i j}= \begin{cases}\left(\frac{i-1}{k_{0}}\right)^{2} & \text { if } i=j, i+j \neq 2 k_{0}+2, \\ 1+e_{0} & \text { if } i=j, i+j=2 k_{0}+2, \\ e_{0} & \text { if } i-j= \pm 2 k_{0} \text { and } i+j \neq 2 k_{0}+2, \\ e_{0} & \text { if } i+j=2 k_{0}+2 \text { and } i-j \neq \pm 2 k_{0}, i \neq j \\ 2 e_{0} & \text { if } i-j= \pm 2 k_{0} \text { and } i+j=2 k_{0}+2 .\end{cases}
$$

It can be seen that Problem 4 is more complex than Problem 3.

\section{Corrections and Asymptotic Behavior of Problems 1 and 2}

The bases in Eq. (9) can be divided into two types:

$$
\begin{aligned}
& \phi_{2 k-1}(x)=\sqrt{\frac{2}{\ell}} \sin \frac{(2 k-1) \pi x}{\ell}, \phi_{2 k-1}(x)=\phi_{2 k-1}(\ell-x), \\
& \phi_{2 k}(x)=\sqrt{\frac{2}{\ell}} \sin \frac{2 k \pi x}{\ell}, \phi_{2 k}(x)=-\phi_{2 k}(\ell-x) .
\end{aligned}
$$

While the former one is symmetric (even function), the latter one is skew-symmetric (odd function).

For Problems 1 and 2, if we insert Eqs. (19) and (20) into Eq. (13), we can derive two diagonal matrices, denoted by

$$
a_{j k}^{o}:=(4 j-2)^{2} \delta_{j k}, a_{j k}^{e}:=16 j^{2} \delta_{j k},
$$

which are independent to $e_{0}$. The eigenvalues derived from the above two diagonal matrices are very simple, given as follows:

$$
\begin{aligned}
& \lambda_{j}^{o}=(4 j-2)^{2}, j=1,2, \ldots, \\
& \lambda_{j}^{e}=(4 j)^{2}, j=1,2, \ldots
\end{aligned}
$$

By arranging them as follows:

$$
\left\{4,16,36,64, \ldots,(4 n-2)^{2},(4 n)^{2}, \ldots\right\}=\left\{(2 i)^{2}, i=1,2,3,4, \ldots\right\},
$$

they are indeed the eigenvalues of the coefficient matrix $\mathbf{A}=\left[a_{i j}\right]$ in Eq. (14) with $e_{0}=0$. They are asymptotically approaching to the true eigenvalues of $\mathbf{A}=\left[a_{i j}\right]$ in Eq. (14).

Lemma 1 For Problem 1 the determinant of the coefficient matrix, denoted by $\mathbf{A}_{n}, n \geq 3$, satisfies the following recursion formula:

$$
\operatorname{Det} \mathbf{A}_{n}=a_{n n} \operatorname{Det} \mathbf{A}_{n-1}-e_{0}^{2} \operatorname{Det} \mathbf{A}_{n-2},
$$

where $\mathbf{A}_{n-1}=\left[a_{i j}\right], i, j=1, \ldots, n-1$ is an $(n-1) \times(n-1)$ sub-matrix of $\mathbf{A}_{n}$, and $\mathbf{A}_{n-2}=\left[a_{i j}\right], i, j=1, \ldots, n-2$ is an $(n-2) \times(n-2)$ sub-matrix of $\mathbf{A}_{n}$. 
Proof. Let us apply the cofactor method to the following determinant:

$$
\begin{aligned}
& \operatorname{Det}_{n}=\left|\begin{array}{ccccccccc}
a_{11} & e_{0} & 0 & 0 & \ldots & 0 & 0 & 0 & 0 \\
e_{0} & a_{22} & e_{0} & 0 & \ldots & 0 & 0 & 0 & 0 \\
\vdots & \vdots & \vdots & \vdots & \ldots & \vdots & \vdots & \vdots & \vdots \\
0 & 0 & 0 & 0 & \ldots & e_{0} & a_{n-2, n-2} & e_{0} & 0 \\
0 & 0 & 0 & 0 & \ldots & 0 & e_{0} & a_{n-1, n-1} & e_{0} \\
0 & 0 & 0 & 0 & \ldots & 0 & 0 & e_{0} & a_{n n}
\end{array}\right| \\
& =a_{n n}\left|\begin{array}{cccccccc}
a_{11} & e_{0} & 0 & 0 & \ldots & 0 & 0 & 0 \\
e_{0} & a_{22} & e_{0} & 0 & \ldots & 0 & 0 & 0 \\
\vdots & \vdots & \vdots & \vdots & \ldots & \vdots & \vdots & \vdots \\
0 & 0 & 0 & 0 & \ldots & e_{0} & a_{n-2, n-2} & e_{0} \\
0 & 0 & 0 & 0 & \ldots & 0 & e_{0} & a_{n-1, n-1}
\end{array}\right| \\
& -e_{0}\left|\begin{array}{cccccccc}
a_{11} & e_{0} & 0 & 0 & \ldots & 0 & 0 & 0 \\
e_{0} & a_{22} & e_{0} & 0 & \ldots & 0 & 0 & 0 \\
\vdots & \vdots & \vdots & \vdots & \ldots & \vdots & \vdots & \vdots \\
0 & 0 & 0 & 0 & \ldots & e_{0} & a_{n-2, n-2} & 0 \\
0 & 0 & 0 & 0 & \ldots & 0 & e_{0} & e_{0}
\end{array}\right| \\
& =a_{n n} \operatorname{Det}_{n-1}-e_{0}^{2}\left|\begin{array}{ccccccc}
a_{11} & e_{0} & 0 & 0 & \ldots & 0 & 0 \\
e_{0} & a_{22} & e_{0} & 0 & \ldots & 0 & 0 \\
\vdots & \vdots & \vdots & \vdots & \ldots & \vdots & \vdots \\
0 & 0 & 0 & 0 & \ldots & e_{0} & a_{n-2, n-2}
\end{array}\right| \\
& =a_{n n} \operatorname{Det} \mathbf{A}_{n-1}-e_{0}^{2} \operatorname{Det} \mathbf{A}_{n-2} .
\end{aligned}
$$

For problem 1 the coefficient matrix $\mathbf{A}$ is a special type Jacobi matrix, whose recursion formula is already known.

In order to find the eigenvalues, we consider the following characteristic equation:

$$
F(\lambda):=\operatorname{Det}\left(\mathbf{A}_{n}-\lambda \mathbf{I}_{n}\right)=0,
$$

where $\mathbf{I}_{n}$ is the $n$-order identity matrix. By using the recursion formula (25), it is easy to compute $F(\lambda)$. The fictitious time integration method (FTIM) developed by Liu and Atluri (2008) used to solve Eq. (27) is summarized as follows.

(i) Give $n, e_{0}, \varepsilon, v, u_{0}, N$, and $s_{0}$,

(ii) Do $j=1, \ldots, n$,

(iii) Do $k=1, \ldots, N$,

$$
\begin{aligned}
& t_{k}=\frac{k-1}{|F(0)|}, \\
& u_{k}=u_{k-1}-(-1)^{j} \frac{v}{\left(1+t_{k}\right)|F(0)|} F\left(u_{k-1}\right),
\end{aligned}
$$

if $\left|F\left(u_{k}\right)\right|<\varepsilon$ then $\lambda_{j}=u_{k}$, set $u_{0}=\lambda_{j}+s_{0}$ and go to (ii); otherwise go to (iii).

For Problem $1, e_{0}$ brings some influence on the lower order eigenvalues. In Table 1, we list the first six eigenvalues computed from the FTIM for $e_{0}=5$.

Table 1. For Problem 1 with $e_{0}=5$, the first six eigenvalues

\begin{tabular}{l|l|l|l|l|l}
\hline$\lambda_{1}$ & $\lambda_{2}$ & $\lambda_{3}$ & $\lambda_{4}$ & $\lambda_{5}$ & $\lambda_{6}$ \\
\hline 2.09946 & 16.6482 & 36.3589 & 64.1989 & 100.1297 & 137.2587 \\
\hline
\end{tabular}

For problem 2, we have the recursion formula for the characteristic function $F(\lambda)$ :

$$
F(\lambda)=-\lambda D(m)-2 e_{0}^{2} E(m),
$$


where $m=n-1$, and $D(m)$ and $E(m)$ are generated from

$$
\begin{aligned}
& D(1)=4-\lambda, \\
& D(2)=(4-\lambda)(16-\lambda)-e_{0}^{2}, \\
& \text { Do } i=3, m, \\
& D(i)=\left[(2 i)^{2}-\lambda\right] D(i-1)-e_{0}^{2} D(i-2),
\end{aligned}
$$

Enddo.

$$
\begin{aligned}
& E(2)=16-\lambda, \\
& E(3)=(16-\lambda)(36-\lambda)-e_{0}^{2}, \\
& \text { Do } i=4, m, \\
& E(i)=\left[(2 i)^{2}-\lambda\right] E(i-1)-e_{0}^{2} E(i-2),
\end{aligned}
$$

Enddo.

In Table 2, we list the first six eigenvalues computed from the FTIM for $e_{0}=1$.

Table 2. For Problem 2 with $e_{0}=1$, the first six eigenvalues

\begin{tabular}{l|l|l|l|l|l}
\hline$\lambda_{1}$ & $\lambda_{2}$ & $\lambda_{3}$ & $\lambda_{4}$ & $\lambda_{5}$ & $\lambda_{6}$ \\
\hline-0.455139 & 4.371301 & 16.033832 & 36.014290 & 64.007947 & 100.02776 \\
\hline
\end{tabular}

The eigenvalues listed are close to that obtained in (McLachlan, 1947; Shivakumar, Williams \& Rudraiah, 1987).

\section{Product Formula for Problems 3 and 4}

By observing Eqs. (17) and (18), we have the coefficient matrix $\mathbf{A}=\left[a_{i j}\right], a_{i j}=0$ if $i+j$ is an odd integer. For example, for Eq. (17) with $k_{0}=1$ and $n=8$, the matrix A looks like

$$
\mathbf{A}=\left[\begin{array}{cccccccc}
a_{11} & 0 & e_{0} & 0 & 0 & 0 & 0 & 0 \\
0 & a_{22} & 0 & e_{0} & 0 & 0 & 0 & 0 \\
e_{0} & 0 & a_{33} & 0 & e_{0} & 0 & 0 & 0 \\
0 & e_{0} & 0 & a_{44} & 0 & e_{0} & 0 & 0 \\
0 & 0 & e_{0} & 0 & a_{55} & 0 & e_{0} & 0 \\
0 & 0 & 0 & e_{0} & 0 & a_{66} & 0 & e_{0} \\
0 & 0 & 0 & 0 & e_{0} & 0 & a_{77} & 0 \\
0 & 0 & 0 & 0 & 0 & e_{0} & 0 & a_{88}
\end{array}\right] .
$$

Furthermore, the characteristic equation can be factored out to be

$$
\begin{gathered}
\operatorname{Det}\left(\mathbf{A}-\lambda \mathbf{I}_{n}\right)=\left|\begin{array}{ccccccccc}
a_{11}-\lambda & 0 & e_{0} & 0 & 0 & 0 & 0 & 0 \\
0 & a_{22}-\lambda & 0 & e_{0} & 0 & 0 & 0 & 0 \\
e_{0} & 0 & a_{33}-\lambda & 0 & e_{0} & 0 & 0 & 0 \\
0 & e_{0} & 0 & a_{44}-\lambda & 0 & e_{0} & 0 & 0 \\
0 & 0 & e_{0} & 0 & a_{55}-\lambda & 0 & e_{0} & 0 \\
0 & 0 & 0 & e_{0} & 0 & a_{66}-\lambda & 0 & e_{0} \\
0 & 0 & 0 & 0 & e_{0} & 0 & a_{77}-\lambda & 0 \\
0 & 0 & 0 & 0 & 0 & e_{0} & 0 & a_{88}-\lambda
\end{array}\right| \\
=\left|\begin{array}{cccc|cccc}
a_{11}-\lambda & e_{0} & 0 & 0 & a_{22}-\lambda & e_{0} & 0 & 0 \\
e_{0} & a_{33}-\lambda & e_{0} & 0 \\
0 & e_{0} & a_{55}-\lambda & e_{0} \\
0 & 0 & e_{0} & a_{77}-\lambda
\end{array}\right| \begin{array}{ccccc}
e_{44}-\lambda & e_{0} & 0 \\
0 & e_{0} & a_{66}-\lambda & e_{0} \\
0 & 0 & e_{0} & a_{88}-\lambda
\end{array} \mid=0 .
\end{gathered}
$$

Now we are ready to prove a major result; however, before that we give two Definitions.

Definition 1 A sub-matrix $\mathbf{B}^{o}$ is said to be an odd-positioned matrix, if all its elements are taken from the coefficient matrix $\mathbf{A}$ at the ijth entry with both $i$ and $j$ being odd integer. Because $i+j$ is an even integer, the elements of $\mathbf{B}^{o}$ are non-zero. 
Definition 2 A sub-matrix $\mathbf{B}^{e}$ is said to be an even-positioned matrix, if all its elements are taken from the coefficient matrix $\mathbf{A}$ at the $i j$ th entry with both $i$ and $j$ being even integer. Because $i+j$ is an even integer, the elements of $\mathbf{B}^{e}$ are non-zero.

Theorem 1 For the coefficient matrix $\mathbf{A}=\left[a_{i j}\right], a_{i j}=0$ if $i+j$ is an odd integer, the characteristic equation of $\mathbf{A}$ can be decomposed into two factors:

$$
\operatorname{Det}\left(\mathbf{A}-\lambda \mathbf{I}_{n}\right)=\operatorname{Det}\left(\mathbf{B}^{o}-\lambda \mathbf{I}_{m_{1}}\right) \times \operatorname{Det}\left(\mathbf{B}^{e}-\lambda \mathbf{I}_{m_{2}}\right)=0
$$

where $m_{1}=m_{2}=n / 2$ if $n$ is an even integer, $m_{1}=(n+1) / 2, m_{2}=(n-1) / 2$ if $n$ is an odd integer, and $m_{1}+m_{2}=n$.

Proof. For $n=8$ we have shown the product equation in the above. For larger $n$, a tedious work can complete the proof.

Theorem 1 is significant, which can greatly simplify the works to find the eigenvalues of symmetric Mathieu's equation. The odd numbered eigenvalues are solved from

$$
\operatorname{Det}\left(\mathbf{B}^{o}-\lambda \mathbf{I}_{m_{1}}\right)=0
$$

while the even numbered eigenvalues are solved from

$$
\operatorname{Det}\left(\mathbf{B}^{e}-\lambda \mathbf{I}_{m_{2}}\right)=0
$$

\section{Eigenvalues Clustering Phenomenon for Problem 3}

\subsection{Problem 3 With $k_{0}=1$}

For Problem 3 with $k_{0}=1$, the asymptotic eigenvalues are very simple, given as follows:

$$
\begin{aligned}
\lambda_{j}^{o} & =(2 j-1)^{2}, j=1,2, \ldots, \\
\lambda_{j}^{e} & =(2 j)^{2}, j=1,2, \ldots
\end{aligned}
$$

By arranging them as follows:

$$
\left\{\lambda_{1}, \lambda_{2}, \lambda_{3}, \lambda_{4}, 25,36,49,64, \ldots,(2 n-1)^{2},(2 n)^{2}, \ldots\right\}=\left\{i^{2}, i=1,2,3,4, \ldots\right\},
$$

they are indeed the eigenvalues of the coefficient matrix $\mathbf{A}=\left[a_{i j}\right]$ in Eq. (16) with $e_{0}=0$. They are asymptotically approaching to the true eigenvalues of $\mathbf{A}=\left[a_{i j}\right]$ in Eq. (18) with $k_{0}=1$.

However, $e_{0}$ must bring some influence on the true eigenvalues. We can correct the lower orders eigenvalues by solving the following characteristic equations:

$$
\begin{aligned}
& \operatorname{Det}\left(\mathbf{B}_{m_{1}}^{o}-\lambda \mathbf{I}_{m_{1}}\right) \\
& =\left|\begin{array}{ccccccccc}
a_{11}-\lambda & e_{0} & 0 & 0 & \ldots & 0 & 0 & 0 & 0 \\
e_{0} & a_{33}-\lambda & e_{0} & 0 & \ldots & 0 & 0 & 0 & 0 \\
\vdots & \vdots & \vdots & \vdots & \ldots & \vdots & \vdots & \vdots & \vdots \\
0 & 0 & 0 & 0 & \ldots & e_{0} & a_{2 m_{1}-5,2 m_{1}-5}-\lambda & e_{0} & 0 \\
0 & 0 & 0 & 0 & \ldots & 0 & e_{0} & a_{2 m_{1}-3,2 m_{1}-3}-\lambda & e_{0} \\
0 & 0 & 0 & 0 & \ldots & 0 & 0 & e_{0} & a_{2 m_{1}-1,2 m_{1}-1}-\lambda
\end{array}\right|=0 \text {, } \\
& \operatorname{Det}\left(\mathbf{B}_{m_{2}}^{e}-\lambda \mathbf{I}_{m_{2}}\right) \\
& =\left|\begin{array}{ccccccccc}
a_{22}-\lambda & e_{0} & 0 & 0 & \ldots & 0 & 0 & 0 & 0 \\
e_{0} & a_{44}-\lambda & e_{0} & 0 & \ldots & 0 & 0 & 0 & 0 \\
\vdots & \vdots & \vdots & \vdots & \ldots & \vdots & \vdots & \vdots & \vdots \\
0 & 0 & 0 & 0 & \ldots & e_{0} & a_{2 m_{2}-4,2 m_{2}-4}-\lambda & e_{0} & 0 \\
0 & 0 & 0 & 0 & \ldots & 0 & e_{0} & a_{2 m_{2}-2,2 m_{2}-2}-\lambda & e_{0} \\
0 & 0 & 0 & 0 & \ldots & 0 & 0 & e_{0} & a_{2 m_{2}, 2 m_{2}}-\lambda
\end{array}\right|=0 .
\end{aligned}
$$

These equations are similar to that in Eq. (26), and thus the same recursion formula (25) can be applied to compute the characteristic functions.

Now, based on the product formula we can apply the FTIM to solve the odd characteristic equation (37) and the even characteristic equation (38), separately. In Table 3, we list the first ten eigenvalues computed from the FTIM for $e_{0}=5$. 
Table 3. For Problem 3 with $k_{0}=1$ and $e_{0}=5$, the first ten eigenvalues

\begin{tabular}{l|l|l|l|l|l|l|l|l|l}
\hline$\lambda_{1}$ & $\lambda_{2}$ & $\lambda_{3}$ & $\lambda_{4}$ & $\lambda_{5}$ & $\lambda_{6}$ & $\lambda_{7}$ & $\lambda_{8}$ & $\lambda_{9}$ & $\lambda_{10}$ \\
\hline-5.7901 & 2.0995 & 9.2363 & 16.6482 & 25.5109 & 36.3589 & 49.2696 & 64.2047 & 81.7732 & 100.6887 \\
\hline
\end{tabular}

6.2 Problem 3 With $k_{0}=2,3$

Problem 3 with $k_{0} \geq 2$ are more complex. By observing Eqs. (17) and (18), the asymptotic eigenvalues are given by

$$
\lim _{n \rightarrow \infty} \lambda_{n}=\left(\frac{n}{k_{0}}\right)^{2}
$$

For lower orders eigenvalues the above formula must be corrected to include the influence from $e_{0}$. Before giving the corrections of lower orders eigenvalues, we can prove the following results.

Theorem 2 For Problem 3 with $k_{0} \geq 2$, the eigenvalues of the coefficient matrix $\mathbf{A}$ with dimension $n=2 k_{0}$ must include $1-e_{0}$ and 4 , and $1-e_{0}$ is the smallest eigenvalue when $e_{0}>1$.

Proof. In Eq. (17) the bands due to $i-j= \pm 2 k_{0}$ are off-diagonal bands, while that due to $i+j=2 k_{0}$ is a cross band. For $\mathbf{A}$ with dimension $n=2 k_{0}$ the elements at the last column and last row are all zeros besides $a_{n n}$. The two reasons are that the off-diagonal bands do not appear not until the dimension $n \geq 2 k_{0}+1$, and the cross band starts from the position $(i, j)=(n-1,1)$ and terminates at the position $(i, j)=(1, n-1)$ due to the constraint $i+j=2 k_{0}=n$. The intersection position of the cross band and the diagonal band is $(i, j)=\left(k_{0}, k_{0}\right)$. Therefore, the elements at the $k_{0}$ th column and the $k_{0}$ th row are all zeros besides $a_{k_{0} k_{0}}$. By the cofactor method we can conclude that there are two eigenvalues $a_{k_{0} k_{0}}=1-e_{0}$ and $a_{n n}=\left(n / k_{0}\right)^{2}=\left(2 k_{0} / k_{0}\right)^{2}=4$ by viewing Eq. (17). Because $1-e_{0}<\left(1 / k_{0}\right)^{2}=a_{11}, 1-e_{0}$ is the smallest eigenvalue.

Theorem 3 For Problem 3 with $k_{0} \geq 2, \lambda=4$ is a common eigenvalue of all the coefficient matrices $\mathbf{A}$ with dimension $4 k_{0}>n \geq 2 k_{0}$.

Proof. The case with $n=2 k_{0}$ was proven in Theorem 2 . We only consider the cases with $n \geq 2 k_{0}+1$, from which the two off-diagonal bands immense. Because $\mathbf{A}$ is symmetric, we only need to look the end position $(i, j)=\left(1,2 k_{0}-1\right)$ of the cross band and the starting position $(i, j)=\left(1,2 k_{0}+1\right)$ of the upper off-diagonal band. Owing to $2 k_{0}-1<2 k_{0}+1$, these two bands have no intersection point. At the gap $2 k_{0}$, the whole column (similarly the whole row) is filled with zero elements, besides the diagonal element $a_{2 k_{0}, 2 k_{0}}$, which has the value $\left(2 k_{0} / k_{0}\right)^{2}=4$ in view of Eqs. (17) and (18). Then, by using the cofactor method we assure that there exists an eigenvalue $\lambda=4$. The intersection of the $i=2 k_{0}$ row with the upper off-diagonal band is happened at $j-i=2 k_{0}$, such that $j=4 k_{0}$. If $n \geq 4 k_{0}, 4$ is not an eigenvalue. Hence, we take $n<4 k_{0}$.

With the help from Theorems 1-3, now for Problem 3, we can carry out the corrections of the first six eigenvalues for $k_{0}=2$ and the corrections of the first eight eigenvalues for $k_{0}=3$. First we discuss $k_{0}=2$ and $n=2 k_{0}=4$, of which $\mathbf{A}$ is given by

$$
\mathbf{A}=\left[\begin{array}{cccc}
\frac{1}{4} & 0 & -e_{0} & 0 \\
0 & 1-e_{0} & 0 & 0 \\
-e_{0} & 0 & \frac{9}{4} & 0 \\
0 & 0 & 0 & 4
\end{array}\right]
$$

If we take $e_{0}=2$, the eigenvalues are found to be

$$
\left(\lambda_{1}, \lambda_{2}, \lambda_{3}, \lambda_{4}\right)=\left(-1, \frac{5}{4}-\sqrt{1+e_{0}^{2}}, \frac{5}{4}+\sqrt{1+e_{0}^{2}}, 4\right)=(-1,-0.98607,3.48607,4) .
$$

We can see that the second eigenvalue is close to the first one. Next we discuss $k_{0}=2$ and $n=2 k_{0}+2=6$, of which $\mathbf{A}$ is given by

$$
\mathbf{A}=\left[\begin{array}{cccccc}
\frac{1}{4} & 0 & -e_{0} & 0 & e_{0} & 0 \\
0 & 1-e_{0} & 0 & 0 & 0 & e_{0} \\
-e_{0} & 0 & \frac{9}{4} & 0 & 0 & 0 \\
0 & 0 & 0 & 4 & 0 & 0 \\
e_{0} & 0 & 0 & 0 & \frac{25}{4} & 0 \\
0 & e_{0} & 0 & 0 & 0 & 9
\end{array}\right]
$$

The off-diagonal bands appear. 4 is still an eigenvalue, but $1-e_{0}$ is no more an eigenvalue. By using Theorem 1 and the Cardano formula, we can find the closed-form solutions of all eigenvalues:

$$
\begin{aligned}
& \left(\lambda_{1}, \lambda_{2}, \lambda_{3}, \lambda_{4}, \lambda_{5}, \lambda_{6}\right) \\
& =(-1.385165,-1.3772,3.19137,4,6.93585,9.385165) .
\end{aligned}
$$


The smallest eigenvalue shifts to -1.385165 .

In Table 4, we list the first ten eigenvalues computed from the FTIM for $e_{0}=2$.

Table 4. For Problem 3 with $k_{0}=2$ and $e_{0}=2$, the first ten eigenvalues

\begin{tabular}{l|l|l|l|l|l|l|l|l|l}
\hline$\lambda_{1}$ & $\lambda_{2}$ & $\lambda_{3}$ & $\lambda_{4}$ & $\lambda_{5}$ & $\lambda_{6}$ & $\lambda_{7}$ & $\lambda_{8}$ & $\lambda_{9}$ & $\lambda_{10}$ \\
\hline-1.4541 & -1.3907 & 2.8572 & 3.6754 & 6.6653 & 9.1422 & 12.6476 & 16.3246 & 21.1072 & 23.4643 \\
\hline
\end{tabular}

Next we consider the corrections of the first eight eigenvalues for $k_{0}=3, n=8$ and $e_{0}=2$, of which we have

$$
\mathbf{A}=\left[\begin{array}{cccccccc}
\frac{1}{9} & 0 & 0 & 0 & -e_{0} & 0 & e_{0} & 0 \\
0 & \frac{4}{9} & 0 & -e_{0} & 0 & 0 & 0 & e_{0} \\
0 & 0 & 1-e_{0} & 0 & 0 & 0 & 0 & 0 \\
0 & -e_{0} & 0 & \frac{16}{9} & 0 & 0 & 0 & 0 \\
-e_{0} & 0 & 0 & 0 & \frac{25}{9} & 0 & 0 & 0 \\
0 & 0 & 0 & 0 & 0 & 4 & 0 & 0 \\
e_{0} & 0 & 0 & 0 & 0 & 0 & \frac{49}{9} & 0 \\
0 & e_{0} & 0 & 0 & 0 & 0 & 0 & \frac{64}{9}
\end{array}\right] .
$$

The off-diagonal bands appear. $1-e_{0}$ and 4 are eigenvalues. By using Theorem 1 and the Cardano formula, we can find the closed-form solutions of all eigenvalues:

$$
\begin{aligned}
& \left(\lambda_{1}, \lambda_{2}, \lambda_{3}, \lambda_{4}, \lambda_{5}, \lambda_{6}, \lambda_{7}, \lambda_{8}\right) \\
& =(-1.42342,-1.32084,-1,2.93694,3.5096,4,6.24716,7.71723) .
\end{aligned}
$$

In Table 5, we list the first ten eigenvalues computed from the FTIM for $e_{0}=2$, where the first three eigenvalues are close. Table 5. For Problem 3 with $k_{0}=3$ and $e_{0}=2$, the first ten eigenvalues

\begin{tabular}{l|l|l|l|l|l|l|l|l|l}
\hline$\lambda_{1}$ & $\lambda_{2}$ & $\lambda_{3}$ & $\lambda_{4}$ & $\lambda_{5}$ & $\lambda_{6}$ & $\lambda_{7}$ & $\lambda_{8}$ & $\lambda_{9}$ & $\lambda_{10}$ \\
\hline-1.4235 & -1.4161 & -1.3851 & 2.6144 & 3.5096 & 4 & 6.2472 & 7.7081 & 9.3852 & 11.5381 \\
\hline
\end{tabular}

\subsection{Clustering Behavior of Eigenvalues}

In order to further explore the clustering behavior of the eigenvalues for Problem 3, we take a $6 \times 6$ sub-matrix of $\mathbf{A}$ in Eq. (44), which is the coefficient matrix for the case $k_{0}=3$ and $n=6$. The six eigenvalues are obtained in closed-form:

$$
\begin{aligned}
& \lambda_{1}=1-e_{0}, \quad \lambda_{2}=\frac{1}{2}\left[a_{22}+a_{44}-\sqrt{\left(a_{44}-a_{22}\right)^{2}+4 e_{0}^{2}}\right], \\
& \lambda_{3}=\frac{1}{2}\left[a_{11}+a_{55}-\sqrt{\left(a_{55}-a_{11}\right)^{2}+4 e_{0}^{2}}\right], \lambda_{4}=\frac{1}{2}\left[a_{22}+a_{44}+\sqrt{\left(a_{44}-a_{22}\right)^{2}+4 e_{0}^{2}}\right], \\
& \lambda_{5}=\frac{1}{2}\left[a_{11}+a_{55}+\sqrt{\left(a_{55}-a_{11}\right)^{2}+4 e_{0}^{2}}\right], \lambda_{6}=4 .
\end{aligned}
$$

When we take $e_{0}=2$, they are

$$
\begin{aligned}
& \left(\lambda_{1}, \lambda_{2}, \lambda_{3}, \lambda_{4}, \lambda_{5}, \lambda_{6}\right) \\
& =(-1,-0.997074,-0.95926,3.219296,3.848145,4) .
\end{aligned}
$$

It can be seen that the first three eigenvalues $(-1,-0.997074,-0.95926)$ are clustered.

In Figure 1, we plot the inverses of the characteristic functions for the full matrix $\mathbf{A}$, odd-positioned matrix $\mathbf{B}^{o}$ and evenpositioned matrix $\mathbf{B}^{e}$. Because the first three eigenvalues $(-1,-0.997074,-0.95926)$ are clustered, the first three picks of the curve for the full matrix merge together. However, with the help of the product formula, the eigenvalues are separated into two groups, which are solved from the characteristic equations belong to $\mathbf{B}^{o}$ and $\mathbf{B}^{e}$. Therefore, as shown in Figure 1, we can observe that the peaks are corresponding to the eigenvalues.

The closeness between the first eigenvalue and second eigenvalue prompts us a question that does there exist a certain value of $e_{0}$ for the equality $\lambda_{1}=\lambda_{2}$. 


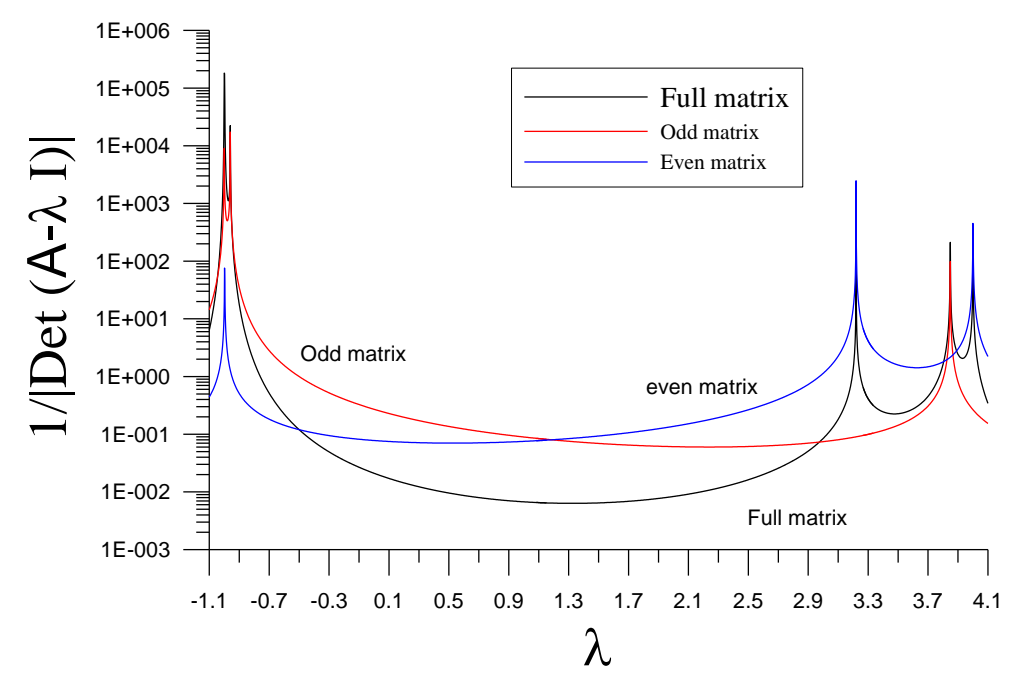

Figure 1. The eigenvalues of Problem 3 with $n=6, k 0=3$ and $e 0=2$. The black line obtained from full matrix indicates the first, second, third, fourth, fifth and sixth eigenvalues, and the odd matrix indicates the first, third and fifth eigenvalues, while the even matrix indicates the second, fourth and sixth eigenvalues

Corollary 1 For Problem 3 with $k_{0}=3$ and $n=6, \lambda_{1}=\lambda_{2}=-17 / 18$ if $e_{0}=35 / 18$.

Proof. From Eq. (46) it follows that

$$
2-2 e_{0}=a_{22}+a_{44}-\sqrt{\left(a_{44}-a_{22}\right)^{2}+4 e_{0}^{2}}
$$

By using $a_{22}=4 / 9$ and $a_{44}=16 / 9$, it yields

$$
2-2 e_{0}=\frac{20}{9}-\sqrt{\frac{16}{9}+4 e_{0}^{2}} .
$$

A further arrangement, leads to

$$
\sqrt{144+324 e_{0}^{2}}=2+18 e_{0}
$$

After taking square on both sides, we have

$$
18 e_{0}=35 .
$$

Hence, both the first eigenvalue and second eigenvalue are

$$
\lambda_{1}=\lambda_{2}=1-e_{0}=-\frac{17}{18} .
$$

For Problem 3, the happening of the double point at real parameter value $e_{0}=35 / 18$, which is close to $e_{0}=2$, is unusual. For Problem 1, the equality $\lambda_{1}=\lambda_{2}$ can occur, only for complex $e_{0}$; the first such example (Meixner, Schäfke \& Wolf, 1980; Blanch \& Clemm, 1969; Shivakumar \& Xue, 1999) happens when $e_{0}=(6.9289547587 \ldots) i$, at which $\lambda_{1}=\lambda_{2}=11.1904735991 \ldots$ For Problem 2 , the equality $\lambda_{1}=\lambda_{2}$ can occur, only for complex $e_{0}$; the first such example (Meixner, Schäfke \& Wolf, 1980; Blanch \& Clemm, 1969) happens when $e_{0}=(1.4687686137 \ldots) i$, at which $\lambda_{1}=\lambda_{2}=2.0886989027 \ldots$

\section{The Eigenvalues of Problem 4}

For Problem 4 the asymptotic eigenvalues are given by

$$
\lim _{n \rightarrow \infty} \lambda_{n}=\left(\frac{n-1}{k_{0}}\right)^{2}
$$


We first discuss $k_{0}=1$ for Problem 4, of which we have the recursion formulas for the characteristic functions $F_{o}(\lambda)$ and $F_{e}(\lambda)$ of $\mathbf{B}_{m_{1}}^{o}$ and $\mathbf{B}_{m_{2}}^{e}$, respectively,

$$
F_{o}(\lambda)=D_{o}\left(m_{1}\right), \quad F_{e}(\lambda)=D_{e}\left(m_{2}\right),
$$

where $D_{o}\left(m_{1}\right)$ and $D_{e}\left(m_{2}\right)$ are generated from

$$
\begin{aligned}
& D_{o}(1)=-\lambda, \\
& D_{o}(2)=\lambda(\lambda-4)-4 e_{0}^{2}, \\
& \text { Do } i=3, m_{1}, \\
& D_{o}(i)=\left[(2(i-1))^{2}-\lambda\right] D_{o}(i-1)-e_{0}^{2} D_{o}(i-2), \\
& \text { Enddo. } \\
& D_{e}(1)=1+e_{0}-\lambda, \\
& D_{e}(2)=\left(1+e_{0}-\lambda\right)(9-\lambda)-e_{0}^{2}, \\
& \text { Do } i=3, m_{2}, \\
& D_{e}(i)=\left[(2 i-1)^{2}-\lambda\right] D_{e}(i-1)-e_{0}^{2} D_{e}(i-2), \\
& \text { Enddo. }
\end{aligned}
$$

In Table 6, we list the first ten eigenvalues computed from the FTIM for $e_{0}=5$. Table 6. For Problem 4 with $k_{0}=1$ and $e_{0}=5$, the first ten eigenvalues

\begin{tabular}{l|l|l|l|l|l|l|l|l|l}
\hline$\lambda_{1}$ & $\lambda_{2}$ & $\lambda_{3}$ & $\lambda_{4}$ & $\lambda_{5}$ & $\lambda_{6}$ & $\lambda_{7}$ & $\lambda_{8}$ & $\lambda_{9}$ & $\lambda_{10}$ \\
\hline-8.6285 & 1.8583 & 9.6734 & 11.5488 & 17.6986 & 25.5501 & 36.3750 & 49.2696 & 64.8814 & 81.7733 \\
\hline
\end{tabular}

Theorem 4 For Problem 4 with $k_{0} \geq 2$, the eigenvalues of the coefficient matrix $\mathbf{A}$ with dimension $n=2 k_{0}$ must include $1+e_{0}$.

Proof. The proof is similar to that for Theorem 2. In Eq. (18), we can see that $1-e_{0}$ in Theorem 2 is replaced by $1+e_{0}$. But it is not the smallest eigenvalue.

Now, we discuss $k_{0}=2$ and $n=2 k_{0}+2=6$ for the corrections of the first six eigenvalues, of which $\mathbf{A}$ is given by

$$
\mathbf{A}=\left[\begin{array}{cccccc}
0 & 0 & 0 & 0 & 2 e_{0} & 0 \\
0 & \frac{1}{4} & 0 & e_{0} & 0 & e_{0} \\
0 & 0 & 1+e_{0} & 0 & 0 & 0 \\
0 & e_{0} & 0 & \frac{9}{4} & 0 & 0 \\
2 e_{0} & 0 & 0 & 0 & 4 & 0 \\
0 & e_{0} & 0 & 0 & 0 & \frac{25}{4}
\end{array}\right]
$$

The off-diagonal bands appear and $1+e_{0}$ is an eigenvalue. By using Theorem 1 and the Cardano formula, we can find the closed-form solutions of all eigenvalues for $e_{0}=2$ :

$$
\begin{aligned}
& \left(\lambda_{1}, \lambda_{2}, \lambda_{3}, \lambda_{4}, \lambda_{5}, \lambda_{6}\right) \\
& =(-2.47214,-1.37721,3,3.19137,6.47214,6.93585) .
\end{aligned}
$$

Next we consider the corrections of the first eight eigenvalues for $k_{0}=3, n=8$ and $e_{0}=2$, of which we have

$$
\mathbf{A}=\left[\begin{array}{cccccccc}
0 & 0 & 0 & 0 & 0 & 0 & 2 e_{0} & 0 \\
0 & \frac{1}{9} & 0 & 0 & 0 & e_{0} & 0 & e_{0} \\
0 & 0 & \frac{4}{9} & 0 & e_{0} & 0 & 0 & 0 \\
0 & 0 & 0 & 1+e_{0} & 0 & 0 & 0 & 0 \\
0 & 0 & e_{0} & 0 & \frac{16}{9} & 0 & 0 & 0 \\
0 & e_{0} & 0 & 0 & 0 & \frac{25}{9} & 0 & 0 \\
2 e_{0} & 0 & 0 & 0 & 0 & 0 & 4 & 0 \\
0 & e_{0} & 0 & 0 & 0 & 0 & 0 & \frac{49}{9}
\end{array}\right]
$$

The off-diagonal bands appear, and $1+e_{0}$ is an eigenvalue. By using Theorem 1 and the Cardano formula, we can find the closed-form solutions of all eigenvalues:

$$
\begin{aligned}
& \left(\lambda_{1}, \lambda_{2}, \lambda_{3}, \lambda_{4}, \lambda_{5}, \lambda_{6}, \lambda_{7}, \lambda_{8}\right) \\
& =(-2.47214,-1.42342,-0.99707,3,3.219296,3.509597,6.24716,6.47214) .
\end{aligned}
$$


For this case the FTIM also leads to the same eigenvalues with very small errors.

In Table 7, we list the first ten eigenvalues computed from the FTIM for $e_{0}=2$.

Table 7. For Problem 4 with $k_{0}=3$ and $e_{0}=2$, the first ten eigenvalues

\begin{tabular}{l|l|l|l|l|l|l|l|l|l}
\hline$\lambda_{1}$ & $\lambda_{2}$ & $\lambda_{3}$ & $\lambda_{4}$ & $\lambda_{5}$ & $\lambda_{6}$ & $\lambda_{7}$ & $\lambda_{8}$ & $\lambda_{9}$ & $\lambda_{10}$ \\
\hline-2.4721 & -1.4234 & -1.3208 & 2.3944 & 2.9369 & 3.5096 & 6.2472 & 6.4721 & 7.7172 & 9.6056 \\
\hline
\end{tabular}

\section{Conclusions}

Resorting on the orthonormal bases and taking the advantage of the skew-symmetry for Problems 1 and 2 and the symmetry for Problems 3 and 4, the eigenvalues problem of Mathieu's equation is transformed to the matrix eigenvalue problem. When the coefficient matrices for Problems 1 and 2 are tridiagonal, the coefficient matrices for Problems 3 and 4 endow with a special structure of the coefficient matrix: $\mathbf{A}=\left[a_{i j}\right], a_{i j}=0$ if $i+j$ is an odd integer. The odd-positioned sub-matrix and even-positioned sub-matrix of $\mathbf{A}$ were introduced. We have proven that the characteristic equation of $\mathbf{A}$ can be decomposed into two factors which are the characteristic equations for the odd-positioned sub-matrix and the even-positioned sub-matrix. Therefore, the order of the nonlinear characteristic equation is reduced to one half. As a consequence, the lower-order eigenvalues were solved in closed-form owing to the specialty of $\mathbf{A}$. The recursion formulae for Problems 1 and 2 and Problems 3 and 4 with $k_{0}=1$ were developed, by which and together with the product formula we have developed a fictitious time integration method (FTIM) to easily find more eigenvalues. We can conclude that the eigenvalues obtained from the matrix eigenvalue problem are very accurate for Mathieu's equation.

\section{Acknowledgements}

The Fundamental Research Funds for the Central Universities under the Grant Number 2017B05714 for the financial support is highly appreciated.

\section{References}

Bateman, H., \& Erdelyi, A. (1955). Higher Transcendental Functions. McGraw-Hill Book Company, New York.

Blanch, G., \& Clemm, D. S. (1969). The double points of Mathieu's differential equation. Math. Comput., $23,97-108$. https://doi.org/10.1090/S0025-5718-1969-0239727-4

Bujurke, N. M., Salimath, C. S., \& Shiralashetti, S. C. (2008). Computation of eigenvalues and solutions of regular Sturm-Liouville problems using Haar wavelets. J. Comput. Appl. Math., 219, 90-101. https://doi.org/10.1016/j.cam.2007.07.005

Gadella, M., Giacomini, H., \& Lara, L. P. (2015). Periodic analytic approximate solutions for the Mathieu equation. Appl. Math. Comput., 271, 436-445. https://doi.org/10.1016/j.amc.2015.09.018

Gheorghiu, C. I., Hochstenbach, M. E., Plestenjak, B., \& Rommes, J. (2012). Spectral collocation solutions to multiparameter Mathieu's system. Appl. Math. Comput., 218, 11990-12000. https://doi.org/10.1016/j.amc.2012.05.068

Liu, C.-S., \& Atluri, S. N. (2008). A novel time integration method for solving a large system of non-linear algebraic equations. Comput. Model. Eng. Sci., 31, 71-83. http://www.techscience.com/doi/10.3970/cmes.2008.031.071.html

McLachlan, N. W. (1947). Theory and Application of Mathieu Functions. Clarendon, Oxford.

Meixner, J., Schäfke, F. W., \& Wolf, G. (1980). Mathieu Functions and Spheroidal Functions and their Mathematical Foundations. Further Studies. Springer-Verlag, Lect. Notes in Math., 837, 85-88.

Plestenjak, B., Gheorghiu, C. I., \& Hochstenbach, M. E. (2015). Spectral collocation for multiparameter eigenvalue problems arising from separable boundary value problems. J. Comput. Phys., 298, 585-601. https://doi.org/10.1016/j.jcp.2015.06.015

Shivakumar, P. N., Williams, J. J., \& Rudraiah, N. (1987). Eigenvalues for infinite matrices. Linear Alg. Appl., $96,35-63$. https://doi.org/10.1016/0024-3795(87)90335-1

Shivakumar, P. N., \& Xue, J. (1999). On the double points of a Mathieu equation. J. Comput. Appl. Math., 107, $111-125$. https://doi.org/10.1016/S0377-0427(99)00084-9

\section{Copyrights}

Copyright for this article is retained by the author(s), with first publication rights granted to the journal.

This is an open-access article distributed under the terms and conditions of the Creative Commons Attribution license (http://creativecommons.org/licenses/by/4.0/). 